\title{
SMOOTH FOLIATIONS ON HOMOGENEOUS COMPACT KÄHLER MANIFOLDS
}

\author{
FEDERICO LO BIANCO AND JORGE VITÓRIO PEREIRA
}

\begin{abstract}
We study smooth foliations of arbitrary codimension on homogeneous compact Kähler manifolds. We prove that smooth foliations on rational compact homogeneous manifolds are locally trivial fibrations and classify the smooth foliations with all leaves analytically dense on compact homogeneous Kähler manifolds. Both results are builded upon a (rough) structure Theorem for smooth foliations on compact homogeneous Kähler manifolds obtained by comparison of the foliation and the Borel-Remmert decomposition of the ambient.
\end{abstract}

\section{INTRODUCTION}

1.1. Motivation. Smooth codimension one foliations on compact homogeneous manifolds have been studied by Ghys in [7]. When the ambient manifold is a compact homogeneous Kähler manifold, a classification is given in [7, Theorem 1.2]. In particular, if the ambient is a compact complex torus $X$ then the foliation is either a linear on foliation on $X$, or $X$ admits a projection $\pi: X \rightarrow E$ to an elliptic curve and $\mathcal{F}$ is transverse to the general fiber of $\pi$.

In this work we investigate smooth foliations of arbitrary codimension on compact homogeneous Kähler manifolds. At the beginning of our investigations we were aiming at a classification result similar to what we have in codimension one, but soon it became apparent that already in codimension two the situation is considerably more involved. We arrived at the example below, as well as at the other examples presented in Section 5 , after reading [9].

Example 1.1. Let $Y$ be a compact homogeneous Kähler manifold and $\mathcal{G}$ be a one dimensional foliation on $Y$ with isolated singularities. Assume there exists a section $\sigma \in$ $H^{0}\left(Y, T^{*} \mathcal{G}\right)$ which does not vanish on $\operatorname{sing}(\mathcal{G})$. Then if we take an arbitrary compact complex torus $A$ and choose an arbitrary vector field $v$ on $A$ we can define an injective morphism

$$
\begin{aligned}
\pi_{Y}^{*} T \mathcal{G} & \longrightarrow T X=\pi_{Y}^{*} T Y \oplus \pi_{A}^{*} T A \\
w & \longmapsto(w, \sigma(w) v)
\end{aligned}
$$

where $\pi_{Y}$ and $\pi_{A}$ are the projections from $X=Y \times A$ onto $Y$ and $A$ respectively. The image of this morphism defines a smooth foliation $\mathcal{F}$ on the compact homogeneous Kähler manifold $X=Y \times A$ with dynamic/geometry at least as complicated as the $d y$ namic/geometry of $\mathcal{G}$.

In the example above the fact that we started with a dimension one foliation with isolated singularities is not really important. We could use dimension one foliations with

Date: October 10, 2018. 
non-isolated singularities. The important thing to carry out the construction is to have sufficiently many independent sections of $T^{*} \mathcal{G}$ in order to generate a subbundle of $T X$ over $\operatorname{sing}(\mathcal{G})$.

If we start with a foliation $\mathcal{G}$ of dimension at least two, then generalizations of the above example are less obvious since we have to take care of the integrabilty condition.

1.2. Rough structure. Our first result is inspired by a Theorem of Brunella concerning the structure of (singular) codimension one foliations on complex tori, see [3].

Theorem A. Let $\mathcal{F}$ be a smooth foliation on a homogeneous compact Kähler manifold X. Then there exists

(1) a locally trivial fibration $\psi: X \rightarrow X^{\prime}$ onto a homogeneous compact Kähler manifold $X^{\prime}$ with fibers isomorphic to a rational homogeneous manifold; and

(2) a locally trivial fibration $\pi: X^{\prime} \rightarrow Y$ onto a homogeneous compact Kähler manifold with fibers isomorphic to a compact complex torus; and

(3) a foliation $\mathcal{F}^{\prime}$ on $X^{\prime}$

such that

(1) $\mathcal{F}=\psi^{*} \mathcal{F}^{\prime}$; and

(2) $\pi_{*} T \mathcal{F}^{\prime}$ is a locally free sheaf of rank $\operatorname{dim} \mathcal{F}^{\prime}$; and

(3) $\operatorname{det} \pi_{*} N \mathcal{F}^{\prime}$ is ample; and

(4) the dimension of $Y$ is at most the codimension of $\mathcal{F}$.

The first locally trivial fibration $\psi: X \rightarrow X^{\prime}$ has all its fibers contained in the leaves of $\mathcal{F}$. For the second locally trivial fibration $\pi: X^{\prime} \rightarrow Y$ the behavior can be different: the general fiber is not necessarily invariant by $\mathcal{F}^{\prime}$.

The proof of Theorem $\mathrm{A}$ follows from an analysis of the linear system $|\operatorname{det} N \mathcal{F}|$. The smoothness of $\mathcal{F}$ together with the homogeneity of $X$ implies that $|\operatorname{det} N \mathcal{F}|$ is base point free and therefore defines a morphism. A standard factorization result for morphisms from compact homogeneous manifolds together with Borel-Remmert structure Theorem allow us to conclude. Details are given in Section 3 .

1.3. Smooth foliations on rational homogeneous manifolds. An immediate corollary of Theorem $\mathrm{A}$ is the fact that rational homogeneous manifolds only carry trivial foliations.

Corollary B. Smooth foliations on rational homogeneous manifolds are locally trivial fibrations.

It is perhaps worth mentioning that in dimension two the only rational surfaces (not homogeneous a priori) which carry smooth foliations by curves are the Hirzebruch surfaces ( $\mathbb{P}^{1}$ bundles over $\mathbb{P}^{1}$ ) according to [2, Proposition 4]. In higher dimensions we are not aware of examples of smooth foliations on rationally connected manifolds which are not fibrations.

1.4. Minimal smooth foliations. Although Example 1.1 shows that we have a lot of freedom to construct smooth foliations on projective homogeneous manifolds, all the examples constructed along the same guidelines will have invariant compact proper subvarieties: the pre-images of the irreducible components of the singular set of $\mathcal{G}$ under the natural projection $Y \times A \rightarrow Y$.

It seems natural to enquire if this is just a coincidence or a general phenomena. In other terms, what can we say about the smooth foliations on compact Kähler homogeneous manifolds that do not leave proper compact subvarieties invariant? Our second main result 
tells that there are not many possibilities: the foliation is essentially a linear foliation on a compact torus.

Theorem $\mathbf{C}$. Let $\mathcal{F}$ be a smooth foliation on a homogeneous compact Kähler manifold X. If every leaf of $\mathcal{F}$ is analytically dense (meaning it is not contained in any proper compact subvariety) then there exist a locally trivial fibration $\pi: X \rightarrow Y$ with rational fibers onto a complex torus $Y$ and a linear foliation $\mathcal{G}$ on $Y$ such that $\mathcal{F}=\pi^{*} \mathcal{G}$.

The proof relies on our Theorem A Bott's vanishing Theorem, and the study of a natural rational map from $X$ to a certain Grassmannian which is constant along fibers of the projection $X \rightarrow Y$ given by Theorem $\mathrm{A}$ and describes how the restriction of $\mathcal{F}$ to these very same fibers varies.

By the Borel-Remmert theorem, a homogeneous compact Kähler manifold $X$ can be decomposed as a product $R \times T$ of a rational homogeneous complex variety $R$ (a generalized flag variety) times a compact complex torus $T$. It is easy to show that locally trivial fibrations preserve this decomposition, so that the morphism $\psi$ (respectively $\pi$ ) in Theorem A restricts to the identity on the torus component (respectively on the rational component). Analogously, the morphism $\pi$ in Theorem $\mathrm{C}$ is nothing but the projection onto the torus component of the Borel-Remmert decomposition.

1.5. Acknowledgements. This research was carried out while the first author was a visiting student at IMPA. We are grateful to IMPA for providing financial support for such visit, and to Serge Cantat for the suggestion of looking at smooth foliations on compact homogeneous manifolds.

\section{Foliations}

2.1. Foliations as subsheaves of the tangent sheaf. A (singular) foliation $\mathcal{F}$ on a complex manifold $X$ is determined by a coherent subsheaf $T \mathcal{F}$ of $T X$ such that

(1) $T \mathcal{F}$ is involutive (closed under the Lie bracket); and

(2) the quotient $T X / T \mathcal{F}$ is torsion free.

The dimension of $\mathcal{F}$ is the generic rank of $T \mathcal{F}$, and the singular set of $\mathcal{F}$ is the singular set of the sheaf $T X / T \mathcal{F}$. A foliation $\mathcal{F}$ is smooth if, and only if, both $T \mathcal{F}$ and $T X / T \mathcal{F}$ are locally free sheaves.

2.2. Foliations as subsheaves of the cotangent sheaf. Alternatively, we can define a foliation through a coherent subsheaf $N^{*} \mathcal{F}$ of $\Omega_{X}^{1}$ such that

(1) $N^{*} \mathcal{F}$ is integrable $\left(d N^{*} \mathcal{F} \subset N^{*} \mathcal{F} \wedge \Omega_{X}^{1}\right)$; and

(2) the quotient $\Omega_{X}^{1} / N^{*} \mathcal{F}$ is torsion free.

The codimension of $\mathcal{F}$ is the generic rank of $N^{*} \mathcal{F}$. Similarly, a foliation $\mathcal{F}$ is smooth if, and only if, both $N^{*} \mathcal{F}$ and $\Omega_{X}^{1} / N^{*} \mathcal{F}$ are locally free sheaves.

2.3. Foliations and differential forms. If $\mathcal{F}$ is a foliation of codimension $q$ then from the inclusion $N^{*} \mathcal{F} \rightarrow \Omega_{X}^{1}$ we deduce a morphism $\operatorname{det} N^{*} \mathcal{F} \rightarrow \Omega_{X}^{q}$. If we set $\mathcal{L}=$ $\left(\operatorname{det} N^{*} \mathcal{F}\right)^{*}$ we get a $q$-form $\omega \in H^{0}\left(X, \Omega_{X}^{q} \otimes \mathcal{L}\right)$ which defines the foliation $\mathcal{F}$ in the sense that $T \mathcal{F}$ can be recovered as the kernel of the morphism

$$
\begin{aligned}
T X & \longrightarrow \Omega_{X}^{q-1} \otimes \mathcal{L} \\
v & \longmapsto i_{v} \omega .
\end{aligned}
$$

If $\mathcal{F}$ is smooth, all the sheaves that we have defined so far are actually locally free, so that

$$
\mathcal{L}=\operatorname{det}\left(N^{*} \mathcal{F}\right)^{*}=\operatorname{det}(N \mathcal{F}) .
$$


2.4. Bott's vanishing Theorem. Given a smooth foliation $\mathcal{F}$ on a complex manifold $X$, Bott showed how to construct a partial holomorphic connection on the normal bundle of $\mathcal{F}$ and along the tangent bundle of $\mathcal{F}$. His construction goes as follows. Given a germ of vector field $v$ tangent to $\mathcal{F}$ and a germ of section $\sigma$ of $N \mathcal{F}$ we want to be able to differentiate $\sigma$ along $v$. To do it, consider an arbitrary lift $\hat{\sigma}$ of $\sigma$ to $T X$, take the bracket of $v$ with $\hat{\sigma}$ and project the result back to $N \mathcal{F}$. This defines a flat partial connection $\nabla$ on $N \mathcal{F}$, which is nowadays called Bott's partial connection.

Applying Chern-Weyl theory to compute the Chern classes of $N \mathcal{F}$ in terms of a $C^{\infty}$ extension of $\nabla$ to a full connection, Bott proved the following fundamental result.

Theorem 2.1. Let $\mathcal{F}$ be a smooth foliation of codimension $q$ on a complex manifold. Any polynomial of degree at least $q+1$ on the Chern classes of the normal bundle of $\mathcal{F}$ vanishes identically.

For a proof see [1, Proposition 3.27]

\section{ROUGH STRUCTURE OF SMOOTH FOLIATIONS}

3.1. Normal reduction. We start by recalling [7, Corollary 2.2] which we state below as lemma.

Lemma 3.1. Let $G / H$ a compact homogeneous manifold and let $\phi: G / H \rightarrow Z$ be a surjective morphism. Then there exist a subgroup $K \supseteq H$ and a morphism with finite fibers $\psi: G / K \rightarrow Z$ such that $\phi=\psi \circ \pi$, where $\pi: G / H \rightarrow G / K$ is the natural projection.

We will apply this lemma to establish what we call the normal reduction of $\mathcal{F}$.

Proposition 3.2. Let $\mathcal{F}$ be a smooth foliation on a compact homogeneous manifold $X$. Then there exist a projection $\pi: X \rightarrow Y$ of $X$ onto a compact homogeneous manifold $Y$, equivariant with respect to the action of $G=A u t^{0}(X)$, and an ample line bundle $\mathcal{L}^{\prime}$ on $Y$ such that $\mathcal{L}=\pi^{*} \mathcal{L}^{\prime}$. We call $\pi: X \rightarrow Y$ the normal reduction of $\mathcal{F}$.

Proof. Let $N \mathcal{F}$ and $T \mathcal{F}$ be respectively the normal and the tangent bundle of $\mathcal{F}$. We have an exact sequence of sheaves (in this case, since $\mathcal{F}$ is smooth, of vector bundles)

$$
0 \rightarrow T \mathcal{F} \rightarrow T X \rightarrow N \mathcal{F} \rightarrow 0 .
$$

Since $X$ is homogeneous $T X$ is globally generated, thus $N \mathcal{F}$ is as well; in particular the base locus of the line bundle $\mathcal{L}:=\operatorname{det}(N \mathcal{F})$ is empty, and we have a morphism

$$
\phi: X \rightarrow \mathbb{P} H^{0}(X, \mathcal{L})^{\vee} \cong \mathbb{P}^{N} \quad N:=h^{0}(X, \mathcal{L})-1
$$

such that $\phi^{*} \mathcal{O}_{\mathbb{P}^{N}}(1)=\mathcal{L}$. Let $Z$ be the image of $\phi$.

Let $\pi: X \rightarrow Y$ and $\psi: Y \rightarrow Z$ be the maps defined by Lemma 3.1, and $\mathcal{L}^{\prime}:=$ $\psi^{*}\left(\mathcal{O}(1)_{\mid Z}\right)$. Then $\mathcal{L}^{\prime}$ is the pull-back by a finite morphism of an ample line bundle, and is therefore ample. To conclude it suffices to notice that $\mathcal{L}=\phi^{*}\left(\mathcal{O}(1)_{\mid Y}\right)=\pi^{*} \mathcal{L}^{\prime}$.

Remark 3.3. Since $\mathcal{L}^{\prime}$ is ample we have that the dimension of $Y$ is given by the formula

$$
\operatorname{dim} Y=\min \left\{n \geq 0 \mid c_{1}(\mathcal{L})^{n+1}=0\right\} .
$$

Bott's vanishing theorem implies the dimension of $Y$ is bounded from above by the codimension of $\mathcal{F}$, i.e. $\operatorname{dim} Y \leq q$. 
3.2. Conormal bundle on fibers of the normal reduction. Let $\mathcal{F}$ be a smooth foliation on a compact homogeneous manifold $X$. Let us consider a fiber $F=\pi^{-1}(q)$ of $\pi$, the normal reduction of $\mathcal{F}$. Notice that $F$ is a compact homogeneous manifold.

Lemma 3.4. There exists a neighbourhood $U \subset Y$ of $q$ such that, if we denote $V=$ $\pi^{-1}(U)$, the sheaf $N^{*} \mathcal{F}_{\mid V}$ is globally generated, i.e. the morphism

$$
H^{0}\left(V, N^{*} \mathcal{F}_{\mid V}\right) \otimes \mathcal{O}_{V} \rightarrow N^{*} \mathcal{F}_{\mid V}
$$

is surjective.

Proof. Let $p$ be a point of $F$ and let $U$ be a neighbourhood of $q$ where $T Y$ and $\mathcal{L}=$ $\pi_{*}(\operatorname{det} N \mathcal{F})$ are both trivial (for example $U$ isomorphic to a product of discs). Then the restriction of $\omega$ to $V=\pi^{-1}(U)$ is a global $q$-form.

In a neighbourhood of $p, \omega$ is decomposable as follows

$$
\omega=\omega_{1} \wedge \ldots \wedge \omega_{q},
$$

where $\omega_{i}=i_{v_{i}} \omega$ for some decomposable local section $v_{i}$ of $\bigwedge^{q-1} T X$. Since $X$ is a homogeneous manifold, $\bigwedge^{q-1} T X$ is globally generated, so we can find global sections $\hat{v}_{1}, \ldots, \hat{v}_{N}$ of $\bigwedge^{q-1} T X$ such that $v_{i}=\sum_{j} \lambda_{i, j} \hat{v}_{j}$ for some local functions $\lambda_{i, j}$.

Now, any local section $\alpha$ of $N^{*} \mathcal{F}$ can be written as $\sum f_{i} \omega_{i}$ for some local functions $f_{i}$. We have then

$$
\alpha=\sum_{i} f_{i} \omega_{i}=\sum_{i, j} f_{i} \lambda_{i, j} i_{\hat{v}_{j}} \omega
$$

which proves the statement since every $i_{\hat{v}_{j}} \omega$ is a global section of $N^{*} \mathcal{F}_{\mid V}$.

The Kähler assumption plays no role in the Lemma above, but it is essential in the result below by Borel and Remmert, see for instance [7, Theorem 2.5].

Theorem 3.5 (Borel-Remmert). Let X be a homogeneous compact Kähler manifold. Then there exists a decomposition

$$
X \cong R \times T
$$

where $R$ is a rational homogeneous projective manifold and $T$ is a complex torus.

3.3. Proof of Theorem $\mathbf{A}$. Let $\mathcal{F}$ be is in the statement of Theorem $\mathrm{A}$ i.e. $\mathcal{F}$ is a smooth foliation on a homogeneous compact Kähler manifold. Let $\pi_{0}: X \rightarrow Y$ be the normal reduction of $\mathcal{F}$, and let

$$
X \cong R \times T, \quad \text { and } \quad Y \cong R^{\prime} \times T^{\prime}
$$

be the Borel-Remmert decompositions of $X$ and $Y$ respectively. The normal reduction of $\mathcal{F}$ respects the product structures of $X$ and $Y$; in other words, there exist two surjective morphisms $\pi_{R}: R \rightarrow R^{\prime}$ and $\pi_{T}: T \rightarrow T^{\prime}$ such that $\pi_{0}=\pi_{R} \times \pi_{T}$. Define

$$
X^{\prime}=R^{\prime} \times T
$$

and let $\psi=\pi_{R} \times i d_{T}$ and $\pi=i d_{R^{\prime}} \times \pi_{T}$, so that $\pi \circ \psi=\pi_{0}$.

Now, by Lemma 3.4 the vector bundle $N^{*} \mathcal{F}$ is globally generated around each fiber of $\pi_{0}$. Let $\alpha$ be a global section of $N^{*} \mathcal{F}$ around a fiber of $\pi_{0}$, that we can see as a global holomorphic 1-form at a neighborhood of this fiber. The fibers of $\psi$ are rational homogeneous manifolds, and since there exist no global holomorphic 1-form on such manifolds, we have that the pull-back of $\alpha$ to any fiber of $\psi$ is identically zero. Therefore the fibers of $\psi$ are contained in leaves of $\mathcal{F}$, and consequently there exists a foliation $\mathcal{F}^{\prime}$ on $X^{\prime}$ such that $\mathcal{F}=\psi^{*} \mathcal{F}^{\prime}$. 
Now let us prove that $\pi_{*} T \mathcal{F}^{\prime}$ is a locally free sheaf of rank equal to $\operatorname{dim} \mathcal{F}$. Let $U \subset Y$ be a sufficiently small open neighbourhood of a point $q \in Y$; then $T X^{\prime}$ is trivial when restricted to $V:=\pi^{-1}(U)$. A section $s$ of $\pi_{*} T \mathcal{F}^{\prime}$ on $U$ is by definition a section of $T \mathcal{F}^{\prime}$ on $V$, so in particular it is a local section of $T X$ on $V$. Since the fibers are parallelizable and compact, the restriction of $s$ to $F:=\pi^{-1}(q)$ is a constant vector field. The claim easily follows by writing the section in local coordinates on the base and global coordinates on the fiber.

By the properties of the normal reduction, we have $\operatorname{det} N \mathcal{F}=\left(\pi^{\prime}\right)^{*} \mathcal{L}$ for some ample line bundle $\mathcal{L}$ on $Y$. On the other hand

$$
\operatorname{det}(N \mathcal{F})=\psi^{*} \operatorname{det}\left(N \mathcal{F}^{\prime}\right)=\psi^{*} \pi^{*} \operatorname{det}\left(\pi_{*} N \mathcal{F}^{\prime}\right)=\left(\pi_{0}\right)^{*} \operatorname{det}\left(\pi_{*} N \mathcal{F}^{\prime}\right),
$$

where the second equality follows from the fact that $T Y$ (and thus $N \mathcal{F}^{\prime}$ and $\operatorname{det} N \mathcal{F}^{\prime}$ ) is globally generated around the fibers of $\pi$. This shows that $\operatorname{det} \pi_{*} N \mathcal{F}^{\prime}$ is an ample linebundle.

Finally the bound on the dimension of $Y$ follows from Remark 3.3

3.4. Proof of Corollary $\mathbb{B}$. Let $\mathcal{F}$ be a smooth foliation on a homogeneous rational manifold $X$; apply Theorem $\mathrm{A}$ to $X$. Since the torus component of $X$ is trivial, we must have $\pi=i d_{X^{\prime}}$. So $\mathcal{F}=\psi^{*} \mathcal{F}^{\prime}$ for some smooth foliation $\mathcal{F}^{\prime}$ such that $\operatorname{det} N \mathcal{F}^{\prime}$ is ample. By Bott's vanishing theorem we have $c_{1}\left(\operatorname{det} N \mathcal{F}^{\prime}\right)^{q+1}=0$ where $q$ is the codimension of $\mathcal{F}^{\prime}$; since $\operatorname{det} N \mathcal{F}^{\prime}$ is ample we obtain $q=\operatorname{dim} X^{\prime}$, so that $\mathcal{F}^{\prime}$ is the foliation by points and $\mathcal{F}$ is the locally trivial fibration $\psi$.

\section{Foliations With All Leaves analytically DENSE}

Throughout this Section we will suppose that the fibers of the normal reduction are tori.

\subsection{Direct image of the tangent bundle.}

Lemma 4.1. Let $\mathcal{F}$ be a smooth foliation on a compact homogeneous manifold $X$. If $\pi: X \rightarrow Y$ is the normal reduction and the fibers of $\pi$ are parallelizable, then the image $\mathcal{T}$ of the natural morphism $\phi: \pi_{*} T \mathcal{F} \rightarrow T Y$ is an involutive subsheaf of $T Y$.

Proof. Let $v, w$ be two local sections of $\mathcal{T}$ on $U \subset Y$. Up to restricting $U$, we can suppose that $v=\phi\left(v_{0}\right), w=\phi\left(w_{0}\right)$ for some local sections $v_{0}, w_{0}$ of $\pi_{*} T \mathcal{F}$, and we can identify $v_{0}$ and $w_{0}$ with sections of $T \mathcal{F}$ on $V:=\pi^{-1}(U)$. Let $x_{1}, \ldots, x_{n}$ be local coordinates on the base and $y_{1}, \ldots y_{m}$ be global coordinates on the (universal cover of the) fiber. Then we can write

$$
v_{0}(x)=\sum_{i} a_{i}(x) \frac{\partial}{\partial x_{i}}+\sum_{j} b_{j}(x) \frac{\partial}{\partial y_{j}}
$$

for some holomorphic functions $a_{i}, b_{j}$ on $\pi^{-1}(U)$. Then

$$
\phi\left(v_{0}\right)=\sum_{i} a_{i}(x) \frac{\partial}{\partial x_{i}}
$$

by writing $w$ in the same way and using that $\frac{\partial}{\partial y_{k}} a_{i}=\frac{\partial}{\partial y_{k}} b_{j}=0$ for any choice of $i, j, k$ we deduce that $\phi$ commutes with Lie brackets. The lemma follows from the involutivity of $T \mathcal{F}$.

Remark 4.2. According to Theorem $\mathrm{A}$, the sheaf $\pi_{*} T \mathcal{F}$ is locally free. But beware that this not imply that $\mathcal{T}$ is a locally free subsheaf of $T Y$. Moreover, even when $\mathcal{T}$ is locally free, it is not necessarily the tangent sheaf of a foliation since $T Y / \mathcal{T}$ is not necessarily torsion free. 
Lemma 4.3. If $\mathcal{T}$ is an involutive subsheaf of $T Y$ then the singular locus of $\mathcal{T}$ is $\mathcal{T}$ invariant.

Proof. Since the claim is local it suffices to prove it locally around $q \in Y$. Let $r$ be the generic rank of $\mathcal{T}$ and $v_{1}, \ldots, v_{r}$ be local sections of $\mathcal{T}$ such that $\psi=v_{1} \wedge \cdots \wedge v_{r}$ is a non-zero local section of $\bigwedge^{r} T Y$. We can write $\psi$ as $h \psi_{0}$ where $h \in \mathcal{O}_{Y, q}$ and $\psi_{0}$ is a local section of $\bigwedge^{r} T Y$ with zero locus of codimension at least two. Since the rank of $\mathcal{T}$ is $r$, it follows that the image of every section of $\bigwedge^{r} \mathcal{T}$ in $\bigwedge^{r} T Y$ is a multiple of $\psi_{0}$. We have then a morphism of $\mathcal{O}_{Y}$-modules

$$
\alpha: \bigwedge^{r} \mathcal{T} \rightarrow \mathcal{O}_{Y}
$$

that maps a local section $\psi=v_{1} \wedge \ldots \wedge v_{r}$ to the local section $f$ of $\mathcal{O}_{Y}$ satisfying $\psi=f \cdot \psi_{0}$. The singular locus of $\mathcal{T}$ is defined by the ideal $\alpha\left(\bigwedge^{r} \mathcal{T}\right)$ which is nothing but the $r$-th Fitting ideal of $\mathcal{T}$, see [6, Section 20.2].

Let $\theta=v_{1} \wedge \cdots \wedge v_{r}$ be a local section of $\wedge^{r} \mathcal{T}$ and $v$ a local section of $\mathcal{T}$. We want to show that $v(\alpha(\theta))$ is contained in the image of $\alpha$. We first calculate the Lie derivative of $\theta:=v_{1} \wedge \ldots \wedge v_{r}$ along $v$ :

$$
L_{v}(\theta)=\left[v, v_{1} \wedge \ldots \wedge v_{r}\right]=\sum_{i=1}^{n}(-1)^{i+1}\left[v, v_{i}\right] \wedge v_{1} \wedge \ldots \wedge v_{i-1} \wedge v_{i+1} \wedge \ldots \wedge v_{r}
$$

where [,] denotes the Schouten-Nijenhuis bracket, which generalises the Lie bracket on the exterior product $\bigwedge^{r} T Y$. Since $v \in \mathcal{T}$ and $\mathcal{T}$ is involutive, we have $\left[v, v_{i}\right] \in \mathcal{T}$, so that $L_{v}\left(v_{1} \wedge \ldots \wedge v_{r}\right)$ is a local section of $\wedge^{r} \mathcal{T}$.

On the other hand

$$
L_{v}(\theta)=\left[v, \alpha(\theta) \psi_{0}\right]=\alpha(\theta)\left[v, \psi_{0}\right]+v(\alpha(\theta)) \psi_{0} .
$$

Now, $L_{v}(\theta)$ belongs to $\bigwedge^{r} \mathcal{T}$. Furthermore, for any choice of a non-zero section $\theta$ of $\bigwedge^{r} \mathcal{T}$, we have

$$
\left[v, \psi_{0}\right]=\frac{L_{v}(\theta)-v(\alpha(\theta)) \psi_{0}}{\alpha(\theta)}=f \cdot \psi_{0}
$$

for some meromorphic function $f$. Since $\left[v, \psi_{0}\right]$ is a holomorphic function, the singular locus of $f$ must be contained in the zero locus of $\psi_{0}$; since the zero locus of $\psi_{0}$ has codimension at least 2 , we conclude that $\left[v, \psi_{0}\right]$ is a multiple of $\psi_{0}$, say $\left[v, \psi_{0}\right]=h \psi_{0}$.

Therefore we can write

$$
\alpha\left(L_{v}(\theta)\right) \psi_{0}=\alpha(\theta) h \psi_{0}+v(\alpha(\theta)) \psi_{0},
$$

and conclude that

$$
v(\alpha(\theta))=\alpha\left(L_{v}(\theta)\right)-\alpha(\theta) h \in \operatorname{Im}(\alpha)
$$

as wanted.

Lemma 4.4. Let $\mathcal{T}$ be a locally free involutive subsheaf of $T Y$. Then the singular locus of $T Y / \mathcal{T}$ is $\mathcal{T}$-invariant.

Proof. Since the claim is local it suffices to prove it locally around $q \in Y$. Let $r$ be the rank of $\mathcal{T}$ and $v_{1}, \ldots, v_{r}$ be generators of $\mathcal{T}$ at a neighborhood of $q$. The singular locus of $T Y / \mathcal{T}$ is defined by the ideal $I$ generated by the coefficients of $\theta=v_{1} \wedge \ldots \wedge v_{r}$.

Let $v$ be a local section of $\mathcal{T} \subset T Y$. In the proof of Lemma 4.3 we have learned that $L_{v}(\theta)$ is a local section of $\bigwedge^{r} \mathcal{T}$. As such it can be written as a multiple of $\theta$, i.e. $L_{v} \theta=H \theta$ for some holomorphic function $H$. 
Choose now commuting vector fields $\xi_{1}, \ldots, \xi_{n}$ around $q$ which generate $T Y$ at a neighborhood of $q$. Then we can write

$$
\theta=\sum a_{J} \xi_{J}
$$

where $J=\left(j_{1}, \ldots, j_{r}\right)$ and $\xi_{J}=\xi_{j_{1}} \wedge \ldots \wedge \xi_{j_{r}}$. If we compute $L_{v} \theta$ in this basis, we get

$$
L_{v} \theta=\sum_{J} L_{v}\left(a_{J} \xi_{J}\right)=\sum_{J}\left(v\left(a_{J}\right) \xi_{J}+a_{J} L_{v}\left(\xi_{J}\right)\right) .
$$

Since the left hand side is equal to $\sum_{J} H a_{J} \xi_{J}$, it follows that the ideal $I$ generated by the $a_{J}$ 's ( the coefficients of $\theta$ ) is left invariant by $v$, i.e. $v(I) \subset I$.

4.2. First integrals. The restriction of $\operatorname{det}(N \mathcal{F})$ around the fibers of $\pi$ is trivial, so that the foliation induced by $\mathcal{F}$ on fibers of $\pi$ is defined by a global holomorphic 1 -forms. Since the fibers are tori the only global 1 -forms are linear forms, so that $\mathcal{F}$ associates to each fiber $F$ a linear subspace of $T_{0} F$ (where we denote by 0 the identity element for a choice of a group law on $F$ ). If $F=\pi^{-1}(q)$ and the image of $\phi$ has maximal rank at $q$, then the above subspace has $\operatorname{dimension} \operatorname{dim} \mathcal{F}-\operatorname{dim} \mathcal{G}$, where $\mathcal{G}=\pi_{*} \mathcal{F}$ is the (singular) foliation on $Y$ whose leaves are the projections of the leaves of $\mathcal{F}$.

If $X=R \times T$ and $Y=R \times T^{\prime}$ are the Borel-Remmert decompositions of $X$ and $Y$ (recall that we are assuming that the fibers of the normal reduction are tori), then $\pi$ is induced by a projection of tori $\pi_{T}: T \rightarrow T^{\prime}$, which can be supposed to be a group homomorphism. The group law on $T^{\prime}$ allows us to canonically identify every fiber with the fiber over $0 \in T^{\prime}$, and the above construction defines a rational function

$$
f: Y \rightarrow G r(k, \operatorname{dim} F),
$$

where $k=\operatorname{dim} \mathcal{F}-\operatorname{dim} \mathcal{G} ; F$ is any fiber of $\pi$ (so that $\operatorname{dim} F=\operatorname{dim} X-\operatorname{dim} Y$ ); and $\operatorname{Gr}(k, \operatorname{dim} F)$ is the Grassmannian of $k$-planes in $\mathbb{C}^{\operatorname{dim} F}$.

Lemma 4.5. The function $f$ is constant along the leaves of $\mathcal{G}$.

Proof. Let $\mathbb{D} \subset Y$ be a small disc contained in one of the leaves of $\mathcal{G}$. It suffices to prove that $f$ is constant on $\mathbb{D}$.

Let us fix linear coordinates $x_{1}, \ldots x_{k}$ on the universal covering of a fiber and a coordinate $y$ on $\mathbb{D}$. We call $\mathcal{H}$ the foliation induced by $\mathcal{F}$ on $\pi^{-1}(\mathbb{D}) \cong \mathbb{D} \times F$. We are going to prove that the foliation induced by $\mathcal{H}$ on each fiber over $\mathbb{D}$ (which is the same as the foliation induced by $\mathcal{F}$ ) does not depend on the chosen fiber (meaning that $f$ is constant along $\mathbb{D})$.

The foliation $\mathcal{H}$ is defined by some vector fields; up to changing the order of the $x_{i}$ 's we can choose a base $v_{0}, \ldots, v_{h}$ of the space of local sections of $T \mathcal{H}$ of the form

$$
v_{0}=\frac{\partial}{\partial y}+\sum_{j \geq h+1} a_{j}^{(0)}(y) \frac{\partial}{\partial x_{j}}, \quad v_{i}=\frac{\partial}{\partial x_{i}}+\sum_{j \geq h+1} a_{j}^{(i)}(y) \frac{\partial}{\partial x_{j}} \quad i=1, \ldots, h .
$$

Here the $a_{j}^{(i)}$ only depend on $y$ because $\operatorname{det}(N \mathcal{F})$ is trivial around fibers, so that $\mathcal{F}$ is defined by global holomorphic 1 -forms on fibers.

The foliation induced by $\mathcal{H}$ on each fiber is defined by the vector fields $v_{1}, \ldots, v_{h}$. Thus we have to prove that $a_{j}^{(i)}$ is constant for all $i=1, \ldots, h$ and $j \geq h+1$. In order to do that we compute the bracket

$$
\left[v_{0}, v_{i}\right]=\sum_{j \geq h+1} \frac{\partial a_{j}^{(i)}}{\partial y} \frac{\partial}{\partial x_{j}}
$$


By involutivity we must have $\left[v_{0}, v_{i}\right] \in \operatorname{Span}\left(v_{0}, \ldots, v_{h}\right)$, but it is easy to see that then $\left[v_{0}, v_{i}\right]=0$, so that the $a_{j}^{(i)} \mathrm{s}$ are constant along $\mathbb{D}$. This proves the lemma.

Corollary 4.6. If there exists a leaf of $\mathcal{G}$ analytically dense then $f: Y \rightarrow G r(k, \operatorname{dim} F)$ is constant. In particular the conclusion holds if all the leaves of $\mathcal{F}$ are analytically dense.

It follows from Lemma 4.5 that the field of meromorphic first integrals of $\mathcal{G}$, i.e. the subfield of $\mathbb{C}(Y)$ formed by meromorphic functions which are constant along the leaves of $\mathcal{G}$, contains the field of meromorphic functions on the Zariski closure of $f(Y)$.

4.3. Proof of Theorem C. By Theorem $\mathrm{A}$ it suffices to prove that, if the fibers of the normal reduction $\pi: X \rightarrow Y$ are tori and all the leaves of $\mathcal{F}$ are analytically dense, then $X$ is a torus and $\mathcal{F}$ is a linear foliation.

The image $\mathcal{T}$ of $\phi: \pi_{*} T \mathcal{F} \rightarrow T Y$ satisfies the hypothesis of Lemma 4.3. Since $S=\operatorname{sing}(\mathcal{T})$ is $\mathcal{T}$-invariant we see that $\pi^{-1}(S)$ is $\mathcal{F}$-invariant. As we are assuming that every leaf of $\mathcal{F}$ is analytically dense, it follows that $\operatorname{sing}(\mathcal{T})$ is empty and $\mathcal{T}$ satisfies the hypothesis of Lemma 4.4. Analogously, we deduce that $\operatorname{sing}(T Y / \mathcal{T})$ is also empty and consequently $\mathcal{T}$ is the tangent bundle of a smooth foliation $\mathcal{G}$ on $Y$ such that $\mathcal{G}=\pi_{*}(\mathcal{F})$. Since all leaves of $\mathcal{F}$ are dense, all leaves of $\mathcal{G}$ are dense too.

Let us consider the commutative diagram

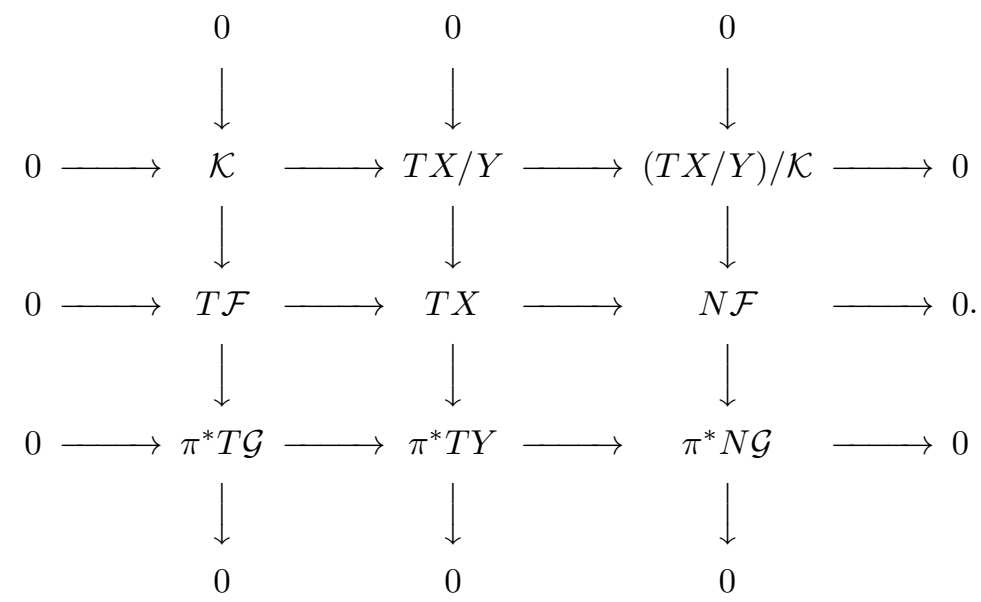

As previously remarked, it follows from Borel-Remmert that $X=R \times T$ and $Y=$ $R \times T^{\prime}$ and the normal reduction morphism $\pi: X \rightarrow Y$ is of the product of the identity over the rational manifold $R$ with a group homomorphism from the torus $T$ to the torus $T^{\prime}$. In particular $T X / Y$ is a free sheaf over $X$.

We will now prove that $\mathcal{K}$ is also a free sheaf. First remark that, since $\mathcal{T}=T \mathcal{G}$ is the tangent sheaf of a smooth foliation, the rank of the sheaf morphism $\widetilde{\phi}: T \mathcal{F} \rightarrow \pi^{*} T \mathcal{G}$ must be maximal at every point, which means that $\mathcal{K}$ is locally free. Moreover it is the tangent sheaf of a smooth foliation $\mathcal{H}$ on $X$ tangent to the fibers of $\pi$ (the intersection between the foliation $\mathcal{F}$ and the fibration $\pi$ ). Now by Corollary 4.6, $T \mathcal{F}$ defines a linear subspace $V \subset T_{0} F$ on each fiber which does not depend on the choice of the fiber; this means that $\mathcal{K}=T \mathcal{H} \cong V \times X$ as desired.

Using the triviality of $\operatorname{det}(T X / Y)$ and of $\operatorname{det}(\mathcal{K})$, we can write

$$
\begin{aligned}
\operatorname{det}(N \mathcal{F}) & =\operatorname{det}\left(\pi^{*} N \mathcal{G}\right) \otimes \operatorname{det}((T X / Y) / \mathcal{K})= \\
& =\pi^{*} \operatorname{det}(N \mathcal{G}) \otimes \operatorname{det}(T X / Y) \otimes \operatorname{det}(\mathcal{K})^{-1}=\pi^{*} \operatorname{det}(N \mathcal{G})
\end{aligned}
$$


By the definition of normal reduction we have that $\operatorname{det}(N \mathcal{G})$ is ample. Bott's vanishing theorem reads as $\operatorname{det}(N \mathcal{G})^{q^{\prime}+1}=0$, where $q^{\prime}$ is the codimension of $\mathcal{G}$. But then $q^{\prime}=$ $\operatorname{dim} Y$, so $\mathcal{G}$ is the foliation by points, and since every leaf of $\mathcal{G}$ is dense we must have $Y=\{p t\}$. It follows that $\operatorname{det}(N \mathcal{F})$ is trivial, so that $\mathcal{F}$ is defined by a global holomorphic $q$-form on $X$. By Borel-Remmert theorem $X$ admits a decomposition

$$
X \cong T \times R
$$

where $T$ is a torus and $R$ is a rational homogeneous manifold. Since there exists no holomorphic global form on $R$, it is easy to see that the subvarieties $\{t\} \times R$ are contained in fibers of $\mathcal{F}$, and thus $X=T$ and $\mathcal{F}$ is defined by a global (i.e. linear) holomorphic form, which proves the theorem.

\section{FURTHER EXAMPLES}

Let $\mathcal{F}$ be a smooth foliation on a compact Kähler homogeneous manifold. Using the notation of Theorem A let $\phi: \pi_{*} T \mathcal{F} \rightarrow T Y$ be the composition of natural morphisms $\pi_{*} T \mathcal{F} \rightarrow \pi_{*} T X \rightarrow T Y$. In general $\phi$ has no reason to be neither injective nor surjective. In the Introduction we already presented examples in which the morphism above is not surjective. For turbulent codimension one foliations on homogeneous manifolds of dimension at least three the morphism $\phi$ is generically surjective but it is not injective.

5.1. Non injective and non generically surjective example. A minor modification of Example 1.1 allow us to construct an example for which the composition $\phi: \pi_{*} T \mathcal{F} \rightarrow T Y$ is not injective nor generically surjective. As before let $\mathcal{G}$ be a one-dimensional foliation on $Y$ with isolated singularities and assume as before that there exists a section $\sigma \in H^{0}\left(Y, T^{*} \mathcal{G}\right)$ which does not vanish on $\operatorname{sing}(\mathcal{G})$. Let $A$ be a compact complex torus of dimension $a$ and let $v_{0}, v_{1}, \ldots, v_{k} \in H^{0}(A, T A)$ be $(k+1)$ linearly independent vector fields on $A(k \leq a-1)$. If $X=Y \times A$ then we have an injection

$$
\begin{aligned}
\left(\pi_{Y}^{*} T \mathcal{G}\right) \oplus \mathcal{O}_{X}{ }^{\oplus k} & \longrightarrow T X=\pi_{Y}^{*} T Y \oplus \pi_{A}^{*} T A \\
\left(w, f_{1}, \ldots, f_{k}\right) & \longmapsto\left(w, \sigma(w) v_{0}+\sum_{i=1}^{k} f_{i} v_{i}\right) .
\end{aligned}
$$

It is a simple matter to verify that the image of the morphism above is an involutive locally free subsheaf of $T X$ of rank $k+1$ with locally free cokernel. Therefore it defines a foliation $\mathcal{F}$ on $X$ of dimension $k+1$, and by construction the image of $\phi: \pi_{*} T \mathcal{F} \rightarrow T Y$ coincides with $T \mathcal{G}$.

5.2. Injective and generically surjective example of arbitrary codimension. Let $Y \subset$ $\mathbb{P}^{N}$ be an abelian variety of dimension $d$; fix $d+1$ hyperplane sections $D_{0}, \ldots D_{d}$ of $Y$ such that $D=\sum D_{i} \in \operatorname{Div}(Y)$ is a simple normal crossing divisor, and the the intersection $D_{0} \cap \ldots \cap D_{d}$ is empty.

Fix another abelian variety $F$ with the same dimension as $Y$. We are going to produce a smooth foliation $\mathcal{F}$ on $X=Y \times F$ such that the image of $\pi_{*} T \mathcal{F}$ in $T Y$ is isomorphic to $T Y(\log D)$; in particular $\phi: \pi_{*} T \mathcal{F} \rightarrow T Y$ is injective and generically surjective, but its image is not the tangent sheaf of a foliation since $T Y / T Y(\log D)$ is not torsion-free.

Lemma 5.1. $\Omega_{Y}^{1}(\log D)$ is globally generated. 
Proof. Let $p \in Y$. Then $p$ belongs to at most $d$ of the $D_{i}$-s, let's say $D_{1}, \ldots, D_{k}$. Let us fix coordinates $x_{1}, \ldots x_{d}$ of $Y$ around $p$ such that $D_{i}=\left\{x_{i}=0\right\}$ for $i=1, \ldots, k$; then the local sections of $\Omega_{Y}^{1}(\log D)$ around $p$ are generated by the forms

$$
\frac{d x_{1}}{x_{1}}, \ldots, \frac{d x_{k}}{x_{k}}, d x_{k+1}, \ldots, d x_{d} .
$$

Now if $l_{0}, \ldots l_{d}$ are linear equations on $\mathbb{P}^{N}$ defining the $D_{i}$-s, then the restriction to $Y$ of the meromorphic form $d \log \left(l_{i} / l_{j}\right)$ defines a global section of $\Omega_{Y}^{1}(\log D)$ whose only poles are along $D_{i}$ and $D_{j}$. In local coordinates around $p$ we have therefore

$$
d \log \left(l_{i} / l_{0}\right)=\lambda_{i} \frac{d x_{i}}{x_{i}}+h o l
$$

for $i=1, \ldots, k$. Since $Y$ is a torus, the sheaf of holomorphic forms $\Omega_{Y}^{1}$ is trivial and in particular globally generated. This proves that each one of the forms $d x_{i} / x_{i}$ can be generated by global sections, which proves the statement.

Any section $\sigma: Y \rightarrow X$ of $\pi: X \rightarrow Y$ defines a foliation $\mathcal{H}$ on $X$ with leaves equal to translations of $\sigma(Y)$ by elements of $F$. The foliation $\mathcal{H}$ induces a natural inclusion of $\pi^{*} T Y(\log D)$ in $T X$, namely

$$
h: \pi^{*} T Y(\log D) \rightarrow T \mathcal{H} \subset T X .
$$

In this way we can see $\pi^{*} T Y(\log D)$ as an involutive subsheaf of $T X$. Note that it does not coincide with the tangent sheaf of $\mathcal{H}$; the cokernel of the inclusion $\pi^{*} T Y(\log D) \rightarrow T \mathcal{H}$ is not torsion-free.

We want to construct a vertical perturbation

$$
v: \pi^{*} T Y(\log D) \rightarrow T X / Y \hookrightarrow T X
$$

in order to define the sheaf $T \mathcal{F}$ as the image of the sum morphism

$$
h+v: \pi^{*} T Y(\log D) \rightarrow T X .
$$

Let $y_{1}, \ldots, y_{l}$ be linear coordinates on the universal covering of $F$. The morphism $v$ can be written as

$$
v=\sum_{i=1}^{l}\left(\pi^{*} s_{i}\right) \frac{\partial}{\partial y_{i}}
$$

for some global sections $s_{1} \ldots, s_{l}$ of $\Omega_{Y}^{1}(\log D)$; reciprocally, every choice of global sections $s_{1}, \ldots, s_{l}$ gives a morphism $v: T Y(\log D) \rightarrow \pi_{*} T X / Y$. We choose global sections $s_{1}, \ldots, s_{l}$ such that on each point of $D$ the image of the vertical morphism defined by the $s_{i}$ is a vector space of maximal dimension $d$; the existence of such sections for a suitable $l$ is assured by Lemma 5.1 .

In order for $T \mathcal{F}$ to be the tangent sheaf of a smooth foliation, we have to verify that: (a) $T \mathcal{F}$ is involutive; $T \mathcal{F}$ is locally free; and $T X / T \mathcal{F}$ is locally free. The fact that $T \mathcal{F}$ and $T X / T \mathcal{F}$ are locally free follows from the definition of $v$.

In order to prove the integrability of $T \mathcal{F}$ take two local sections of $\pi^{*} T Y(\log D)$

$$
v_{1}=\sum_{i=1}^{d} a_{i} \partial_{x_{i}}, \quad v_{2}=\sum_{j=1}^{d} b_{j} \partial_{x_{j}},
$$

where $x_{1}, \ldots x_{d}$ are local coordinates on $Y$ and $\partial_{x_{i}}:=\partial / \partial x_{i}$. We have to check that

$$
\left[(h+v)\left(v_{1}\right) ;(h+v)\left(v_{2}\right)\right] \in \operatorname{Im}(h+v) .
$$


Since $\pi^{*} T Y(\log D)$ is globally generated around fibers of $\pi$, we can assume without loss of generality that $a_{i}$ and $b_{j}$ only depend on the $x$ coordinates (i.e. are constant along the fibers of $\pi$ ). Thus

$$
\left[(h+v)\left(v_{1}\right) ;(h+v)\left(v_{2}\right)\right]=h\left(\left[v_{1} ; v_{2}\right]\right)+\left[h\left(v_{1}\right) ; v\left(v_{2}\right)\right]+\left[v\left(v_{1}\right) ; h\left(v_{2}\right)\right] .
$$

Hence we have to prove that $\left[h\left(v_{1}\right) ; v\left(v_{2}\right)\right]+\left[v\left(v_{1}\right) ; h\left(v_{2}\right)\right]=v\left(\left[v_{1}, v_{2}\right]\right)$. We have

$$
\left[v_{1} ; v_{2}\right]=\sum a_{i} \frac{\partial b_{j}}{\partial x_{i}} \partial_{x_{j}}-\sum b_{j} \frac{\partial a_{i}}{\partial x_{j}} \partial_{x_{i}}=\sum\left(a_{i} \frac{\partial b_{j}}{\partial x_{i}}-b_{i} \frac{\partial a_{j}}{\partial x_{i}}\right) \partial_{x_{j}}
$$

so that

$$
v\left(\left[v_{1} ; v_{2}\right]\right)=\sum\left(a_{i} \frac{\partial b_{j}}{\partial x_{i}}-b_{i} \frac{\partial a_{j}}{\partial x_{i}}\right) s_{k}\left(\partial_{x_{j}}\right) \partial_{y_{k}} .
$$

On the other hand

$$
\left[h\left(v_{1}\right) ; v\left(v_{2}\right)\right]=\sum a_{i} \frac{\partial b_{j}}{\partial x_{i}} s_{k}\left(\partial_{x_{j}}\right) \partial_{y_{k}}+\sum a_{i} b_{j} \frac{\partial}{\partial x_{i}}\left(s_{k}\left(\partial_{x_{j}}\right)\right) \partial_{y_{k}}
$$

so that

$$
\begin{gathered}
{\left[h\left(v_{1}\right) ; v\left(v_{2}\right)\right]+\left[v\left(v_{1}\right) ; h\left(v_{2}\right)\right]=} \\
v\left(\left[v_{1} ; v_{2}\right]\right)+\sum a_{i} b_{j}\left(\frac{\partial}{\partial x_{j}}\left(s_{k}\left(\partial_{x_{i}}\right)\right)-\frac{\partial}{\partial x_{i}}\left(s_{k}\left(\partial_{x_{j}}\right)\right)\right) \partial_{y_{k}} .
\end{gathered}
$$

Since the equality must be true for all choices of $v_{1}$ and $v_{2}$, we now have to prove that for all $i, j, k$

that is $d s_{k}\left(\partial_{x_{i}}, \partial_{x_{j}}\right)=0$.

$$
\frac{\partial}{\partial x_{j}}\left(s_{k}\left(\partial_{x_{i}}\right)\right)-\frac{\partial}{\partial x_{i}}\left(s_{k}\left(\partial_{x_{j}}\right)\right)=0
$$

Since $s_{k}$ is a logarithmic 1-form with normal crossing polar divisor on a compact Kähler manifold, it must be closed by a Theorem of Deligne, see [5, Corollary 3.2.14] for the original proof, and [8] or [4, Lemma 2.1] for short analytic proofs.

\section{REFERENCES}

[1] Paul Baum and Raoul Bott. Singularities of holomorphic foliations. J. Differential Geometry, 7:279-342, 1972.

[2] Marco Brunella. Feuilletages holomorphes sur les surfaces complexes compactes. Ann. Sci. École Norm. Sup. (4), 30(5):569-594, 1997.

[3] Marco Brunella. Codimension one foliations on complex tori. Ann. Fac. Sci. Toulouse Math. (6), 19(2):405418, 2010.

[4] Marco Brunella and Luís Gustavo Mendes. Bounding the degree of solutions to Pfaff equations. Publ. Mat., 44(2):593-604, 2000.

[5] Pierre Deligne. Théorie de Hodge. II. Inst. Hautes Études Sci. Publ. Math., (40):5-57, 1971.

[6] David Eisenbud. Commutative algebra, volume 150 of Graduate Texts in Mathematics. Springer-Verlag, New York, 1995. With a view toward algebraic geometry.

[7] Étienne Ghys. Feuilletages holomorphes de codimension un sur les espaces homogènes complexes. Ann. Fac. Sci. Toulouse Math. (6), 5(3):493-519, 1996.

[8] Junjiro Noguchi. A short analytic proof of closedness of logarithmic forms. Kodai Math. J., 18(2):295-299, 1995.

[9] Ivan Pan and Marcos Sebastiani. Classification des feuilletages turbulents. Ann. Fac. Sci. Toulouse Math. (6), 12(3):395-413, 2003.

I.R.M.A.R., Université de Rennes I, Campus de Beaulieu, 35042 Rennes Cedex, France

E-mail address: federico.1o-bianco@univ-rennes1.fr

IMPA, Estrada Dona CASTORINa, 110, Horto, Rio DE JANEIRo, BRAsiL

E-mail address: jvp@impa.br 